Bull. Korean Math. Soc. 52 (2015), No. 3, pp. 865-880

http://dx.doi.org/10.4134/BKMS.2015.52.3.865

\title{
EXPANDING THE APPLICABILITY OF SECANT METHOD WITH APPLICATIONS
}

\author{
Á. Alberto Magreñán and Ioannis K. Argyros
}

\begin{abstract}
We present new sufficient convergence criteria for the convergence of the secant-method to a locally unique solution of a nonlinear equation in a Banach space. Our idea uses Lipschitz and center-Lipschitz instead of just Lipschitz conditions in the convergence analysis. The new convergence criteria can always be weaker than the corresponding ones in earlier studies. Numerical examples are also provided in this study to solve equations in cases not possible before.
\end{abstract}

\section{Introduction}

In this study we are concerned with the problem of approximating a locally unique solution $x^{\star}$ of equation

$$
F(x)=0,
$$

where $F$ is a Fréchet-differentiable operator defined on a convex subset $\mathcal{D}$ of a Banach space $\mathcal{X}$ with values in a Banach space $\mathcal{Y}$.

A vast number of problems from applied science including engineering can be solved by means of finding the solutions equations in a form like (1.1) using mathematical modelling $[7,11,16,19]$. For example, dynamic systems are mathematically modeled by difference or differential equations, and their solutions usually represent the states of the systems. Except in special cases, the solutions of these equations cannot be found in closed form. This is the main reason why the most commonly used solution methods are iterative. Iteration methods are also applied for solving optimization problems. In such cases, the iteration sequences converge to an optimal solution of the problem at hand. Since all of these methods have the same recursive structure, they can be introduced and discussed in a general framework. The convergence analysis of iterative methods is usually divided into two categories: semilocal and local convergence analysis. In the semilocal convergence analysis one derives convergence criteria from the information around an initial point whereas in

Received March 24, 2014; Revised August 26, 2014.

2010 Mathematics Subject Classification. 47H17, 49M15, 65H10, 65B05, 65G99, 65N30.

Key words and phrases. secant method, Banach space, majorizing sequence, divided difference, Fréchet-derivative. 
the local analysis one finds estimates of the radii of convergence balls from the information around a solution.

We consider the secant method in the form

$$
x_{n+1}=x_{n}-\delta F\left(x_{n-1}, x_{n}\right)^{-1} F\left(x_{n}\right) \quad(n \geq 0), \quad\left(x_{-1}, x_{0} \in \mathcal{D}\right)
$$

where $\delta F(x, y) \in \mathcal{L}(\mathcal{X}, \mathcal{Y})(x, y \in \mathcal{D})$ the space of bounded linear operators from $\mathcal{X}$ into $\mathcal{Y}$ of the Fréchet-derivative of $F[16,19]$.

The semilocal convergence matter is, based on the information around an initial point, to give criteria ensuring the convergence of iteration procedures. A very important problem in the study of iterative procedures is the convergence domain. In general the convergence domain is small. Therefore, it is important to enlarge the convergence domain without additional hypotheses. Another important problem is to find more precise error estimates on the distances $\left\|x_{n+1}-x_{n}\right\|,\left\|x_{n}-x^{\star}\right\|$. These are our objectives in this paper.

The secant method, also known under the name of Regula Falsi or the method of chords, is one of the most used iterative procedures for solving nonlinear equations. According to A. N. Ostrowski [20], this method is known from the time of early Italian algebraists. In the case of equations defined on the real line, the secant method is better than Newton's method from the point of view of the efficiency index [7]. The secant method was extended for the solution of nonlinear equations in Banach Spaces by A. S. Sergeev [25] and J. W. Schmidt [24].

The simplified secant method

$$
x_{n+1}=x_{n}-\delta F\left(x_{-1}, x_{0}\right)^{-1} F\left(x_{n}\right) \quad(n \geq 0), \quad\left(x_{-1}, x_{0} \in D\right)
$$

was first studied by S. Ulm [26]. The first semilocal convergence analysis was given by P. Laasonen [22]. His results was improved by F. A. Potra and V. Pták [21-23]. A semilocal convergence analysis for general secant-type methods was given in general by J. E. Dennis [15]. Bosarge and Falb [10], Dennis [11], Potra [21-23], Argyros [5-9], Hernández et al. [15] and others [14], [19], [27], have provided sufficient convergence conditions for the secant method based on Lipschitz-type conditions on $\delta F$.

The conditions usually associated with the semilocal convergence of secant method (1.2) are:

- $F$ is a nonlinear operator defined on a convex subset $\mathcal{D}$ of a Banach space $\mathcal{X}$ with values in a Banach space $\mathcal{Y}$;

- $x_{-1}$ and $x_{0}$ are two points belonging to the interior $\mathcal{D}^{0}$ of $\mathcal{D}$ and satisfying the inequality

$$
\left\|x_{0}-x_{-1}\right\| \leq c
$$

- $F$ is Fréchet-differentiable on $\mathcal{D}^{0}$, and there exists an operator $\delta F: \mathcal{D}^{0} \times$ $\mathcal{D}^{0} \rightarrow \mathcal{L}(\mathcal{X}, \mathcal{Y})$ such that: 
the linear operator $A=\delta F\left(x_{-1}, x_{0}\right)$ is invertible, its inverse $A^{-1}$ is bounded, and:

$$
\begin{gathered}
\left\|A^{-1} F\left(x_{0}\right)\right\| \leq \eta ; \\
\left\|A\left[\delta F(x, y)-F^{\prime}(z)\right]\right\| \leq \ell(\|x-z\|+\|y-z\|) ;
\end{gathered}
$$

for all $x, y, z \in \mathcal{D}$;

$$
\ell c+2 \sqrt{\ell \eta} \leq 1
$$

The sufficient convergence condition (1.3) is easily violated (see the Numerical Examples). Hence, there is no guarantee in these cases that equation (1.1) under the information $(\ell, c, \eta)$ has a solution that can be found using secant method (1.2). In this study we are motivated by optimization considerations, and the above observation.

The use of Lipschitz and center-Lipschitz conditions is one way used to enlarge the convergence domain of different methods. This technique consists of using both conditions together instead of using only the Lipschitz one which allows us to find a finer majorizing sequence, that is, a larger convergence domain. It has been used in order to find weaker convergence criteria for Newton's method by Argyros in [8]. Gutiérrez et al. in [13] give sufficient conditions for Newton's method using both Lipschitz and center-Lipschitz conditions, Magreñán in [18] for the damped Newton's methods and Amat et al. in [2,4] or García-Olivo [12] for other methods.

Here using Lipschitz and center-Lipschitz conditions, we provide a new semilocal convergence analysis for (1.2). It turns out that our new convergence criteria can always be weaker than the old ones given in earlier studies such as $[1,14,17,19,21-24,27,28]$. The paper is organized as follows: The semilocal convergence analysis of the secant method is presented in Section 2. Numerical examples are provided in Section 3.

\section{Semilocal convergence analysis of the secant method}

In this Section, we present the semilocal convergence analysis of the secantmethod (1.2). First, we present two auxiliary results concerning convergence criteria and majorizing sequences.

Lemma 2.1. Let $\ell_{0}>0, \ell>0, c>0$ and $\eta>0$ be constants with $\ell_{0} \leq \ell$. Then, the following items hold

(i)

$$
0<\frac{\ell(c+\eta)}{1-\ell_{0}(c+\eta)} \leq \frac{2 \ell}{\ell+\sqrt{\ell^{2}+4 \ell_{0} \ell}}<\frac{1-\ell_{0}(c+\eta)}{1-\ell_{0} c} \Leftrightarrow c+\eta \leq \frac{4 \ell^{2}}{\left(\ell+\sqrt{\ell^{2}+4 \ell_{0} \ell}\right)^{2}} ;
$$

$$
\ell c \leq \frac{3-\sqrt{1+4 \frac{\ell_{0}}{\ell}}}{1+\sqrt{1+4 \frac{\ell}{\ell_{0}}}} \Leftrightarrow \frac{(1-\ell c)^{2}}{4} \leq b^{2}-\ell c
$$


(iii)

$$
\ell c \geq \frac{3-\sqrt{1+4 \frac{\ell_{0}}{\ell}}}{1+\sqrt{1+4 \frac{\ell}{\ell_{0}}}} \Leftrightarrow \frac{(1-\ell c)^{2}}{4} \geq b^{2}-\ell c
$$

(iv)

$$
\ell c \leq \frac{3-\sqrt{1+4 \frac{\ell_{0}}{\ell}}}{1+\sqrt{1+4 \frac{\ell}{\ell_{0}}}} \text { and } \ell c+\sqrt{\ell \eta} \leq 1 \Rightarrow c+\eta \leq \frac{4 \ell}{\left(\ell+\sqrt{\ell^{2}+4 \ell_{0} \ell}\right)^{2}} c
$$

(v)

$$
\ell c \geq \frac{3-\sqrt{1+4 \frac{\ell_{0}}{\ell}}}{1+\sqrt{1+4 \frac{\ell}{\ell_{0}}}} \text { and } c+\eta \leq \frac{4 \ell}{\left(\ell+\sqrt{\ell^{2}+4 \ell_{0} \ell}\right)^{2}} \Rightarrow \ell c+\sqrt{\ell \eta} \leq 1 .
$$

Proof. Let $x=1-\ell c, y=\ell \eta, a=\frac{\ell_{0}}{\ell}$ and $b=\frac{2}{1+\sqrt{1+4 a}}$. Then, we have that $a b^{2}+b-1=0$ and $a b+1=\frac{1}{b}$.

(i) The triple inequality in (2.1) holds, if

$$
\begin{gathered}
\frac{\ell c+\ell \eta}{1-a \ell(c+\eta)} \leq \frac{2 \ell}{\ell+\sqrt{\ell^{2}+4 a \ell^{2}}}=b, \\
b<\frac{1-a \ell(c+\eta)}{1-a \ell c}
\end{gathered}
$$

and

$$
\ell(c+\eta)<\frac{1}{a}
$$

or, if

$$
\begin{gathered}
y \leq b^{2}-(1-x), \\
y<\frac{1-b}{a}-(1-b)(1-x)=b^{2}-(1-b)(1-x),
\end{gathered}
$$

and

$$
y \leq \frac{1}{a}-(1-x),
$$

respectively. We have that $a b^{2}=1-b<1$ by the definition of $a$ and $b$. It follows that

$$
b^{2}-(1-x)<\frac{1}{a}-(1-x)
$$

and from $(1-b)(1-x)<(1-x)$ we get that

$$
b^{2}-(1-x)<b^{2}-(1-b)(1-x) .
$$

Hence, it follows from (2.12) and (2.13) that (2.6)-(2.8) are satisfied if (2.9) holds. But (2.9) is equivalent to the right hand side inequality in (2.1). Conversely, if the right hand side inequality in (2.1) holds, then (2.9), (2.12) and 
(2.13) imply (2.10) and (2.11) imply (2.6)-(2.8) which imply the triple inequality in (2.1).

(ii)

$$
\begin{aligned}
\ell c & \leq \frac{3-\sqrt{1+4 \frac{\ell_{0}}{\ell}}}{1+\sqrt{1+4 \frac{\ell}{\ell_{0}}}} \Leftrightarrow 2(1-b)<x<2(1+b) \Leftrightarrow x^{2}-4 x+4\left(1-b^{2}\right) \leq 0 \\
& \Leftrightarrow \frac{x^{2}}{4} \leq b^{2}-(1-x) \Leftrightarrow \frac{(\ell \eta)^{2}}{4} \leq b^{2}-\ell c .
\end{aligned}
$$

(iii)

$$
\begin{aligned}
& \frac{(\ell \eta)^{2}}{4} \geq b^{2}-\ell c \Leftrightarrow \frac{x^{2}}{4} \geq b^{2}-(1-x) \Leftrightarrow x^{2}-4 x+4\left(1-b^{2}\right) \geq 0 \\
& \Rightarrow x \leq 2(1-b) \Leftrightarrow \ell c \geq \frac{3-\sqrt{1+4 \frac{\ell_{0}}{\ell}}}{1+\sqrt{1+4 \frac{\ell}{\ell_{0}}}}
\end{aligned}
$$

(since $x \geq 2(1+b)$ cannot hold).

(iv) The hypotheses in (2.4) and (2.2) imply $\ell \eta \leq b^{2}-\ell c$ which is

$$
c+\eta \leq \frac{4 \ell}{\left(\ell+\sqrt{\ell^{2}+4 \ell_{0} \ell}\right)^{2}} .
$$

(v) The hypothesis in (2.5) and (2.3) imply

$$
\ell c+\sqrt{\ell \eta} \leq 1
$$

We need the following result on majorizing sequences for the secant method (1.2).

Lemma 2.2. Let $\ell_{0}>0, \ell>0, c>0$, and $\eta>0$ be constants with $\ell_{0} \leq \ell$.

Suppose

$$
c+\eta \leq \frac{4 \ell^{2}}{\ell+\sqrt{\ell^{2}+4 \ell_{0} \ell}} .
$$

Then, scalar sequence $\left\{t_{n}\right\}(n \geq-1)$ given by

(2.15) $t_{-1}=0, t_{0}=c, t_{1}=c+\eta, t_{n+2}=t_{n+1}+\frac{\ell\left(t_{n+1}-t_{n-1}\right)\left(t_{n+1}-t_{n}\right)}{1-\ell_{0}\left(t_{n+1}-t_{0}+t_{n}\right)}$

is increasing, bounded from above by

$$
t^{\star \star}=\frac{\eta}{1-b}+c
$$

and converges to its unique least upper bound $t^{\star}$ such that

$$
c+\eta \leq t^{\star} \leq t^{\star \star}
$$

Moreover, the following estimates hold for all $n \geq 0$ :

$$
0 \leq t_{n+2}-t_{n+1} \leq b\left(t_{n+1}-t_{n}\right) \leq b^{n+1} \eta,
$$

where $b$ is given in Lemma 2.1. 
Proof. We shall show using induction on $k \geq 0$ that

$$
0 \leq t_{k+2}-t_{k+1} \leq b\left(t_{k+1}-t_{k}\right)
$$

Using (2.15) for $k=0$, we must show

$$
0<\frac{\ell\left(t_{1}-t_{-1}\right)}{1-\ell_{0} t_{1}} \leq b
$$

or

$$
0<\frac{\ell(c+\eta)}{1-\ell_{0}(c+\eta)} \leq b
$$

which is true by (2.1) and (2.14). Let assume that (2.19) holds for $k \leq n+1$.

It then follows from the induction hypotheses that

$$
\begin{aligned}
t_{k+2} & \leq t_{k+1}+b\left(t_{k+1}-t_{k}\right) \\
& \leq t_{k}+b\left(t_{k}-t_{k-1}\right)+b\left(t_{k+1}-t_{k}\right) \\
& \leq t_{1}+b\left(t_{1}-t_{0}\right)+\cdots+b\left(t_{k+1}-t_{k}\right) \\
& \leq c+\eta+b \eta+\cdots+b^{k+1} \eta \\
& =c+\frac{1-b^{k+2}}{1-b} \eta<\frac{\eta}{1-b}+c=t^{\star \star} .
\end{aligned}
$$

Moreover, we can have:

$$
\begin{aligned}
& \ell\left(t_{k+2}-t_{k+1}\right)+b \ell_{0}\left(t_{k+2}-t_{0}+t_{k+1}\right) \\
\leq & \ell\left(\left(t_{k+2}-t_{k+1}\right)+\left(t_{k+1}-t_{k}\right)\right)+b \ell_{0}\left(\frac{1-b^{k+2}}{1-b}+\frac{1-b^{k+1}}{1-b}\right) \eta+b \ell_{0} c \\
\leq & \ell\left(b^{k}+b^{k+1}\right) \eta+\frac{b \ell_{0}}{1-b}\left(2-b^{k+1}-b^{k+2}\right) \eta+b \ell_{0} c .
\end{aligned}
$$

In view of (2.21), inequality (2.19) holds, if

$$
\ell\left(b^{k}+b^{k+1}\right) \eta+\frac{b \ell_{0}}{1-b}\left(2-b^{k+1}-b^{k+2}\right) \eta+b \ell_{0} c \leq b
$$

or

$(2.23) \ell\left(b^{k-1}+b^{k}\right) \eta+\ell_{0}\left(\left(1+b+\cdots+b^{k}\right)+\left(1+b+\cdots+b^{k+1}\right)\right) \eta+\ell_{0} c-1 \leq 0$.

In view of (2.23), we are motivated to define recurrent functions for $k \geq 1$ on $[0,1)$ by

$$
f_{k}(t)=\ell\left(t^{k-1}+t^{k}\right) \eta+\ell_{0}\left(2\left(1+t+\cdots+t^{k}\right)+t^{k+1}\right) \eta+\ell_{0} c-1
$$


We need the relationship between two consecutive functions $f_{k}$. Using (2.24), we obtain

$$
\begin{aligned}
f_{k+1}(t)= & \ell\left(t^{k}+t^{k+1}\right) \eta+\ell_{0}\left(2\left(1+t+\cdots+t^{k+1}\right)+t^{k+2}\right) \eta+\ell_{0} c-1 \\
= & \ell\left(t^{k-1}+t^{k}\right) \eta+\ell\left(t^{k}+t^{k+1}\right) \eta-\ell\left(t^{k-1}+t^{k}\right) \eta \\
& +\ell_{0}\left(2\left(1+t+\cdots+t^{k}\right)+t^{k+1}\right) \eta+\ell_{0}\left(2 t^{k+1}+t^{k+2}\right) \eta \\
& -\ell_{0} t^{k+1} \eta+\ell_{0} c-1 \\
= & f_{k}(t)+\ell\left(t^{k+1}-t^{k-1}\right) \eta+\ell_{0}\left(t^{k+1}+t^{k+2}\right) \eta \\
= & p(t) t^{k-1} \eta+f_{k}(t),
\end{aligned}
$$

where $p(t)=\ell_{0} t^{3}+\left(\ell_{0}+\ell\right) t^{2}-\ell$. Notice that by Descarte's rule of signs, $b$ is the only positive root of polynomial $p$. We can show instead of (2.23)

$$
f_{k}(b) \leq 0, \quad k \geq 1 \text {. }
$$

Define functions $f_{\infty}$ on interval $[0,1)$ by $f_{\infty}(t)=\lim _{k \rightarrow \infty} f_{k}(t)$. Then, in view of (2.24) we get that

$$
f_{\infty}(t)=\frac{2 \ell_{0} \eta}{1-t}+\ell_{0} c-1
$$

We have that $f_{k}(b)=f_{k+1}(b)=f_{\infty}(b)$. Hence, we can show instead of $(2.26)$ that $f_{\infty}(b) \leq 0$, which is true by $(2.1),(2.14)$ and $(2.27)$. Hence, we showed sequence $\left\{t_{n}\right\} \quad(n \geq-1)$ is increasing and bounded from above by $t^{\star \star}$, so that (2.18) holds. It follows that there exists $t^{\star} \in\left[c+\eta, t^{\star \star}\right]$, so that $\lim _{n \rightarrow \infty} t_{n}=$ $t^{\star}$.

We denote by $U(z, \varrho)$ the open ball centered ar $z \in \mathcal{X}$ and of radius $\varrho>0$. We also denote by $\bar{U}(z, \varrho)$ the closure of $U(z, \varrho)$. We shall study the secant method (1.2) for triplets $\left(F, x_{-1}, x_{0}\right)$ belonging to the class $\mathcal{C}\left(\ell, \ell_{0}, \eta, c\right)$ defined as follows:

Definition 2.3. Let $\ell, \ell_{0}, \eta, c$ be positive constants satisfying the hypotheses of Lemma 2.2 .

We say that a triplet $\left(F, x_{-1}, x_{0}\right)$ belongs to the class $\mathcal{C}\left(\ell, \ell_{0}, \eta, c\right)$ if:

$\left(c_{1}\right) F$ is a nonlinear operator defined on a convex subset $\mathcal{D}$ of a Banach space $\mathcal{X}$ with values in a Banach space $\mathcal{Y}$;

$\left(\mathrm{c}_{2}\right) x_{-1}$ and $x_{0}$ are two points belonging to the interior $\mathcal{D}^{0}$ of $\mathcal{D}$ and satisfying the inequality

$$
\left\|x_{0}-x_{-1}\right\| \leq c ;
$$

(c $\left.c_{3}\right) F$ is Fréchet-differentiable on $\mathcal{D}^{0}$, and there exists an operator $\delta F: \mathcal{D}^{0} \times$ $\mathcal{D}^{0} \rightarrow \mathcal{L}(\mathcal{X}, \mathcal{Y})$ such that: 
the linear operator $A=\delta F\left(x_{-1}, x_{0}\right)$ is invertible, its inverse $A^{-1}$ is bounded and:

$$
\begin{aligned}
\left\|A^{-1} F\left(x_{0}\right)\right\| & \leq \eta ; \\
\left\|A\left[\delta F(x, y)-F^{\prime}(z)\right]\right\| & \leq \ell(\|x-z\|+\|y-z\|) ; \\
\left\|A\left[\delta F(x, y)-F^{\prime}\left(x_{0}\right)\right]\right\| & \leq \ell_{0}\left(\left\|x-x_{0}\right\|+\left\|y-x_{0}\right\|\right)
\end{aligned}
$$

for all $x, y, z \in \mathcal{D}$.

$\left(c_{4}\right)$ the set $\mathcal{D}_{c}=\{x \in \mathcal{D} ; F$ is continuous at $x\}$ contains the closed ball $\bar{U}\left(x_{0}, t^{\star}-t_{0}\right)$, where $t^{\star}$ is given in Lemma 2.2. (1.2).

We present the following semilocal convergence theorem for secant method

Theorem 2.4. If $\left(F, x_{-1}, x_{0}\right) \in \mathcal{C}\left(\ell, \ell_{0}, \eta, c\right)$, then sequence $\left\{x_{n}\right\}(n \geq-1)$ generated by secant method (1.2) is well defined, remains in $\bar{U}\left(x_{0}, t^{\star}-t_{0}\right)$ for all $n \geq 0$ and converges to a unique solution $x^{\star} \in \bar{U}\left(x_{0}, t^{\star}-t_{0}\right)$ of equation $F(x)=0$. Moreover the following estimates hold for all $n \geq 0$

$$
\left\|x_{n+2}-x_{n+1}\right\| \leq t_{n+2}-t_{n+1},
$$

and

$$
\left\|x_{n}-x^{\star}\right\| \leq t^{\star}-t_{n},
$$

where the sequence $\left\{t_{n}\right\}(n \geq 0)$ given by (2.15). Furthermore, if there exists $R \geq t^{\star}-t_{0}$, such that

$$
\ell_{0}\left(c+\frac{\eta}{1-b}+R\right) \leq 1
$$

and

$$
U\left(x_{0}, R\right) \subseteq \mathcal{D},
$$

then the solution $x^{\star}$ is unique in $\bar{U}\left(x_{0}, R\right)$.

Proof. We first show operator $L=\delta F(u, v)$ is invertible for $u, v \in \bar{U}\left(x_{0}, t^{\star}-t_{0}\right)$. It follows from $(2.1),\left(\mathrm{c}_{2}\right)$ and $\left(\mathrm{c}_{3}\right)$ that:

$$
\begin{aligned}
\left\|I-A^{-1} L\right\| & =\left\|A^{-1}(L-A)\right\| \\
& \leq\left\|A^{-1}\left(L-F^{\prime}\left(x_{0}\right)\right)\right\|+\left\|A^{-1}\left(F^{\prime}\left(x_{0}\right)-A\right)\right\| \\
& \leq \ell_{0}\left(\left\|u-x_{0}\right\|+\left\|v-x_{0}\right\|+\left\|x_{0}-x_{-1}\right\|\right) \\
& \leq \ell_{0}\left(t^{\star}-t_{0}+t^{\star}-t_{0}+c\right) \\
& \leq \ell_{0}\left(2\left(\frac{\eta}{1-b}+c\right)-c\right)<1 .
\end{aligned}
$$

According to the Banach Lemma on invertible operators [8], [16], and (2.32), $L$ is invertible and

$$
\left\|L^{-1} A\right\| \leq\left(1-\ell_{0}\left(\left\|x_{k}-x_{0}\right\|+\left\|x_{k+1}-x_{0}\right\|+c\right)\right)^{-1} .
$$


The second condition in $\left(\mathrm{c}_{3}\right)$ implies the Lipschitz condition for $F^{\prime}$

$$
\left\|A^{-1}\left(F^{\prime}(u)-F^{\prime}(v)\right)\right\| \leq 2 \ell\|u-v\|, \quad u, v \in \mathcal{D}^{0} .
$$

By the identity,

$$
F(x)-F(y)=\int_{0}^{1} F^{\prime}(y+t(x-y)) d t(x-y)
$$

we get

(2.36) $\left\|A_{0}^{-1}\left[F(x)-F(y)-F^{\prime}(u)(x-y)\right]\right\| \leq \ell(\|x-u\|+\|y-u\|)\|x-y\|$ and

$$
\begin{aligned}
& \left\|A_{0}^{-1}[F(x)-F(y)-\delta F(u, v)(x-y)]\right\| \\
\leq & \ell(\|x-v\|+\|y-v\|+\|u-v\|)\|x-y\|
\end{aligned}
$$

for all $x, y, u, v \in \mathcal{D}^{0}$. By a continuity argument (2.34)-(2.37) remain valid if $x$ and/or $y$ belong to $\mathcal{D}_{c}$. We first show (2.28). If (2.28) holds for all $n \leq k$ and if $\left\{x_{n}\right\}(n \geq 0)$ is well defined for $n=0,1,2, \ldots, k$ then

$$
\left\|x_{0}-x_{n}\right\| \leq t_{n}-t_{0}<t^{\star}-t_{0}, \quad n \leq k .
$$

That is (1.2) is well defined for $n=k+1$. For $n=-1$, and $n=0,(2.28)$ reduces to $\left\|x_{-1}-x_{0}\right\| \leq c$, and $\left\|x_{0}-x_{1}\right\| \leq \eta$. Suppose (2.28) holds for $n=-1,0,1, \ldots, k(k \geq 0)$. Using (2.33), (2.37) and

$$
F\left(x_{k+1}\right)=F\left(x_{k+1}\right)-F\left(x_{k}\right)-\delta F\left(x_{k-1}, x_{k}\right)\left(x_{k+1}-x_{k}\right)
$$

we obtain in turn:

$$
\begin{aligned}
\left\|A^{-1} F\left(x_{k+1}\right)\right\| & =\ell\left(\left\|x_{k+1}-x_{k}\right\|+\left\|x_{k}-x_{k-1}\right\|\right)\left\|x_{k+1}-x_{k}\right\| \\
& =\ell\left(t_{k+1}-t_{k}+t_{k}-t_{k-1}\right)\left(t_{k+1}-t_{k}\right) \\
& =\ell\left(t_{k+1}-t_{k-1}\right)\left(t_{k+1}-t_{k}\right)
\end{aligned}
$$

and

$$
\begin{aligned}
\left\|x_{k+2}-x_{k+1}\right\| & =\left\|\delta F\left(x_{k}, x_{k+1}\right)^{-1} F\left(x_{k+1}\right)\right\| \\
& \leq\left\|\delta F\left(x_{k}, x_{k+1}\right)^{-1} A\right\|\left\|A^{-1} F\left(x_{k+1}\right)\right\| \\
& \leq \frac{\ell\left(t_{k+1}-t_{k}+t_{k}-t_{k-1}\right)}{1-\ell_{0}\left(t_{k+1}-t_{0}+t_{k}-t_{0}+t_{0}-t_{-1}\right)}\left(t_{k+1}-t_{k}\right) \\
& =t_{k+2}-t_{k+1} .
\end{aligned}
$$

The induction for (2.28) is completed. It follows from (2.28) and Lemma 2.2 that sequence $\left\{x_{n}\right\}(n \geq-1)$ is complete in a Banach space $\mathcal{X}$, and as such it converges to some $x^{\star} \in \bar{U}\left(x_{0}, t^{\star}-t_{0}\right)$ (since $\bar{U}\left(x_{0}, t^{\star}-t_{0}\right)$ is a closed set). By letting $k \rightarrow \infty$ in (2.41), we obtain $F\left(x^{\star}\right)=0$. Estimate (2.29) follows from $(2.28)$ by using standard majoration techniques $[7,16,19,23]$. We shall first show uniqueness in $\bar{U}\left(x_{0}, t^{\star}-t_{0}\right)$. Let $y^{\star} \in \bar{U}\left(x_{0}, t^{\star}-t_{0}\right)$ be a solution of equation (1.1). 
Set

It then by $\left(\mathrm{c}_{3}\right)$ :

$$
\mathcal{M}=\int_{0}^{1} F^{\prime}\left(y^{\star}+t\left(y^{\star}-x^{\star}\right)\right) d t .
$$

$$
\begin{aligned}
\left\|A^{-1}(A-\mathcal{M})\right\| & =\ell_{0}\left(\left\|y^{\star}-x_{0}\right\|+\left\|x^{\star}-x_{0}\right\|+\left\|x_{0}-x_{-1}\right\|\right) \\
& \leq \ell_{0}\left(\left(t^{\star}-t_{0}\right)+\left(t^{\star}-t_{0}\right)+t_{0}\right) \\
& \leq \ell_{0}\left(2\left(\frac{\eta}{1-b}+c\right)-c\right) \\
& =\ell_{0}\left(\frac{2 \eta}{1-b}+c\right)<1 .
\end{aligned}
$$

It follows from (2.1), and the Banach lemma on invertible operators that $\mathcal{M}^{-1}$ exists on $\bar{U}\left(x_{0}, t^{\star}-t_{0}\right)$. Using the identity:

$$
F\left(x^{\star}\right)-F\left(y^{\star}\right)=\mathcal{M}\left(x^{\star}-y^{\star}\right)
$$

we deduce $x^{\star}=y^{\star}$. Finally, we shall show uniqueness in $U\left(x_{0}, R\right)$. As in (2.42), we arrive at

by $(2.30)$.

$$
\left\|A^{-1}(A-\mathcal{M})\right\|<\ell_{0}\left(\frac{\eta}{1-b}+c+R\right) \leq 1,
$$

Remark 2.5. (a) Let us define the majoring sequence $\left\{w_{n}\right\}$ used in earlier studies such as $[1,14,17,19,21-24,27,28]$ (under condition (1.3)):

$w_{-1}=0, w_{0}=c, w_{1}=c+\eta, w_{n+2}=w_{n+1}+\frac{\ell\left(w_{n+1}-w_{n-1}\right)\left(w_{n+1}-w_{n}\right)}{1-\ell\left(w_{n+1}-w_{0}+w_{n}\right)}$.

Note that in general

$$
\ell_{0} \leq \ell
$$

holds, and $\frac{\ell}{\ell_{0}}$ can be arbitrarily large [5-8]. In the case $\ell_{0}=\ell$, then $t_{n}=w_{n}$ $(n \geq-1)$. Otherwise:

$$
\begin{gathered}
t_{n+1}-t_{n} \leq w_{n+1}-w_{n} \\
0 \leq t^{\star}-t_{n} \leq w^{\star}-w_{n}, \quad w^{\star}=\lim _{n \rightarrow \infty} w_{n} .
\end{gathered}
$$

Note also that strict inequality holds in (2.46) for $n \geq 1$, if $\ell_{0}<\ell$. It is worth noticing that the center-Lipschitz condition is not an additional hypothesis to the Lipschitz condition, since in practice the computation of constant $\ell$ requires the computation of $\ell_{0}$. It follows from the proof of Theorem 2.4 that sequence $\left\{s_{n}\right\}$ defined by

$$
\begin{aligned}
& s_{-1}=0, \quad s_{0}=c, \quad s_{1}=c+\eta, \quad s_{2}=s_{1}+\frac{\ell_{0}\left(s_{1}-s_{-1}\right)\left(s_{1}-s_{0}\right)}{1-\ell_{0} s_{1}} \\
& s_{n+2}=s_{n+1}+\frac{\ell\left(s_{n+1}-s_{n-1}\right)\left(s_{n+1}-s_{n}\right)}{1-\ell_{0}\left(s_{n+1}-s_{0}+s_{n}\right)} \quad \text { for } n=1,2, \ldots
\end{aligned}
$$


is also a majorizing sequence for $\left\{x_{n}\right\}$ which is tighter than $\left\{t_{n}\right\}$.

(b) In practice constant $c$ depends on initial guesses $x_{-1}$ and $x_{0}$ which can be chosen to be as close to each other as we wish. Therefore, in particular, we can always choose

$$
\ell c<\frac{3-\sqrt{1+4 \frac{\ell_{0}}{\ell}}}{1+\sqrt{1+4 \frac{\ell_{0}}{\ell}}},
$$

which according to (iv) in Lemma 2.1 implies that the new sufficient convergence criterion (2.14) is weaker than the old one given by (1.3).

\section{Numerical examples}

Example 3.1. Let $\mathcal{X}=\mathcal{Y}=\mathcal{C}[0,1]$, equipped with the max-norm. Consider the following nonlinear boundary value problem

$$
\left\{\begin{array}{c}
u^{\prime \prime}=-u^{3}-\gamma u^{2} \\
u(0)=0, \quad u(1)=1 .
\end{array}\right.
$$

It is well known that this problem can be formulated as the integral equation

$$
u(s)=s+\int_{0}^{1} \mathcal{Q}(s, t)\left(u^{3}(t)+\gamma u^{2}(t)\right) d t,
$$

where $\mathcal{Q}$ is the Green function:

$$
\mathcal{Q}(s, t)= \begin{cases}t(1-s), & t \leq s \\ s(1-t), & s<t .\end{cases}
$$

We observe that

$$
\max _{0 \leq s \leq 1} \int_{0}^{1}|\mathcal{Q}(s, t)| d t=\frac{1}{8} .
$$

Then problem (3.1) is in the form (1.1), where, $F: \mathcal{D} \longrightarrow \mathcal{Y}$ is defined as

$$
[F(x)](s)=x(s)-s-\int_{0}^{1} \mathcal{Q}(s, t)\left(x^{3}(t)+\gamma x^{2}(t)\right) d t .
$$

The Fréchet derivative of the operator $F$ is given by

$$
\left[F^{\prime}(x) y\right](s)=y(s)-3 \int_{0}^{1} \mathcal{Q}(s, t) x^{2}(t) y(t) d t-2 \gamma \int_{0}^{1} \mathcal{Q}(s, t) x(t) y(t) d t .
$$

Then, we have that

$$
\left[\left(I-F^{\prime}\left(x_{0}\right)\right)(y)\right](s)=3 \int_{0}^{1} \mathcal{Q}(s, t) x_{0}^{2}(t) y(t) d t+2 \gamma \int_{0}^{1} \mathcal{Q}(s, t) x_{0}(t) y(t) d t .
$$

Hence, if $2 \gamma<5$, then

$$
\left\|I-F^{\prime}\left(x_{0}\right)\right\| \leq 2(\gamma-2)<1 .
$$

It follows that $F^{\prime}\left(x_{0}\right)^{-1}$ exists and

$$
\left\|F^{\prime}\left(x_{0}\right)^{-1}\right\| \leq \frac{1}{5-2 \gamma} .
$$


We also have that $\left\|F\left(x_{0}\right)\right\| \leq 1+\gamma$. Define the divided difference defined by

$$
\delta F(x, y)=\int_{0}^{1} F^{\prime}(y+t(x-y)) d t .
$$

Choosing $x_{-1}(s)$ such that $\left\|x_{-1}-x_{0}\right\| \leq c$ and $k_{0} c<1$. Then, we have

$$
\left\|\delta F\left(x_{-1}, x_{0}\right)^{-1} F\left(x_{0}\right)\right\| \leq\left\|\delta F\left(x_{-1}, x_{0}\right)^{-1} F^{\prime}\left(x_{0}\right)\right\|\left\|F^{\prime}\left(x_{0}\right) F\left(x_{0}\right)\right\|
$$

and

$$
\left\|\delta F\left(x_{-1}, x_{0}\right)^{-1} F^{\prime}\left(x_{0}\right)\right\| \leq \frac{1}{\left(1-k_{0} c\right)}
$$

where $k_{0}$ is such that

$$
\left\|F^{\prime}\left(x_{0}\right)^{-1}\left(F^{\prime}\left(x_{0}\right)-A_{0}\right)\right\| \leq k_{0} c .
$$

Set $u_{0}(s)=s$ and $\mathcal{D}=U\left(u_{0}, R\right)$. It is easy to verify that $U\left(u_{0}, R\right) \subset U(0, R+1)$ since $\left\|u_{0}\right\|=1$. If $2 \gamma<5$, and $k_{0} c<1$ the operator $F^{\prime}$ satisfies conditions of Theorem 2.6, with

$$
\eta=\frac{1+\gamma}{\left(1-k_{0} c\right)(5-2 \gamma)}, \quad l=\frac{\gamma+6 R+3}{8(5-2 \gamma)\left(1-k_{0} c\right)}, \quad l_{0}=\frac{2 \gamma+3 R+6}{16(5-2 \gamma)\left(1-k_{0} c\right)} .
$$

Choosing $R_{0}=0.9, \gamma=0.5$ and $c=1$ we obtain that

$$
\begin{gathered}
k_{0}=0.1938137822 \cdots, \\
\eta=0.465153 \cdots, \\
l=0.344989 \cdots
\end{gathered}
$$

and

$$
l_{0}=0.187999 \cdots .
$$

Then, criterion (1.3) is not satisfied since $l c+2 \sqrt{l \eta}=1.14617 \cdots>1$, but criterion (2.14) is satisfied since

$$
\eta+c=1.46515 \cdots \leq \frac{4 l}{\left(l^{2}+\sqrt{l^{2}+4 l_{0} l}\right)^{2}}=1.49682 \cdots .
$$

As a consequence the convergence of the secant-method is guaranteed by Theorem 2.4.

Example 3.2. Let $\mathcal{X}=\mathcal{Y}=\mathbb{R}$ and let consider the real functions

$$
F(x)=x^{3}-k,
$$

where $k \in \mathbb{R}$ and we are going to apply secant-method to find the solution of $F(x)=0$. We take the starting point $x_{0}=1$ we consider the domain $\Omega=B\left(x_{0}, 1\right)$ and we let $x_{-1}$ free in order to find a relation between $k$ and $x_{-1}$ for which criterion (1.3) is not satisfied but new criterion (2.14) is satisfied. In this case, we obtain

$$
\begin{aligned}
& \eta=\left|(1-k)\left(1+x_{-1}+x_{-1}^{2}\right)\right|, \\
& l=\frac{6}{\left|1+x_{-1}+x_{-1}^{2}\right|}
\end{aligned}
$$




$$
l_{0}=\frac{9}{2\left|1+x_{-1}+x_{-1}^{2}\right|} .
$$

Taking all this data into account we obtain the following criteria:

(i) If $55 / 54<k \leq 25 / 24$ and

$$
\alpha<x_{-1} \leq \frac{2-27 k}{2(-29+27 k)}-\frac{1}{2} \sqrt{3} \sqrt{-\frac{2164-3024 k+729 k^{2}}{(-29+27 k)^{2}}},
$$

where $\alpha$ is the smallest positive root of

$p(t)-73+24 k+(22+48 k) t+(-111+72 k) t^{2}+(-38+48 k) t^{3}+(-25+24 k) t^{4}$.

(ii) If $25 / 24<k<29 / 27$ and

$$
1<x_{1} \leq \frac{2-27 k}{2(-29+27 k)}-\frac{1}{2} \sqrt{3} \sqrt{-\frac{2164-3024 k+729 k^{2}}{(-29+27 k)^{2}}} .
$$

(iii) If $55 / 54<k<25 / 24$ and

$$
\frac{56-27 k}{2(-29+27 k)}+\frac{1}{2} \sqrt{3} \sqrt{-\frac{-968-108 k+729 k^{2}}{(-29+27 k)^{2}}} \leq x_{-1}<\alpha,
$$

where $\alpha$ is the greatest positive root of $p(t)=-49+24 k+(22+48 k) t+(-111+72 k) t^{2}+(-62+48 k) t^{3}+(-25+24 k) t^{4}$.

(iv) If $25 / 24 \leq k<29 / 27$ and

$$
\frac{56-27 k}{2(-29+27 k)}+\frac{1}{2} \sqrt{3} \sqrt{-\frac{-968-108 k+729 k^{2}}{(-29+27 k)^{2}}} \leq x_{-1}<1 .
$$

(v) If $25 / 27<k<23 / 24$ and

$$
1 \leq x_{-1}<\frac{52-27 k}{2(-25+27 k)}-\frac{1}{2} \sqrt{3} \sqrt{-\frac{-968+108 k+729 k^{2}}{(-25+27 k)^{2}}} .
$$

(vi) If $23 / 24 \leq k<53 / 54$ and

$$
\alpha \leq x_{-1}<\frac{52-27 k}{2(-25+27 k)}-\frac{1}{2} \sqrt{3} \sqrt{-\frac{-968+108 k+729 k^{2}}{(-25+27 k)^{2}}},
$$

where $\alpha$ is the smallest positive root of $p(t)=25+24 k+(-118+48 k) t+(-33+72 k) t^{2}+(-58+48 k) t^{3}+(-23+24 k) t^{4}$.

(vii) If $25 / 27<k \leq 23 / 24$ and

$$
\frac{-2-27 k}{2(-25+27 k)}+\frac{1}{2} \sqrt{3} \sqrt{-\frac{1732-2808 k+729 k^{2}}{(-25+27 k)^{2}}} \leq x_{-1}<1 .
$$


(viii) If $23 / 24<k<53 / 54$ and

$$
\frac{-2-27 k}{2(-25+27 k)}+\frac{1}{2} \sqrt{3} \sqrt{-\frac{1732-2808 k+729 k^{2}}{(-25+27 k)^{2}}} \leq x_{-1}<\alpha,
$$

where $\alpha$ is the greatest positive root of

$p(t)=1+24 k+(-118+48 k) t+(-33+72 k) t^{2}+(-34+48 k) t^{3}+(-23+24 k) t^{4}$.

Now we consider a case in which both criteria (1.3) and (2.14) are satisfied to compare the majorizing sequences. We choose $k=0.99$ and $x_{-1}=1.2$ and we obtain

$$
c=0.2, \quad \eta=0.0364 \cdots, \quad l=1.64835, \quad l_{0}=1.23626 .
$$

Moreover, criterion (1.3)

$$
l c+2 \sqrt{l \eta}=0.819568<1,
$$

is satisfied and criterion (2.14)

$$
c+\eta=0.2364 \cdots \leq 0.26963 \cdots=\frac{4 l}{\left(l^{2}+\sqrt{l^{2}+4 l_{0} l}\right)^{2}},
$$

is also satisfied. In Table 1 it is shown that $\left\{s_{n}\right\},\left\{t_{n}\right\}$ and $\left\{w_{n}\right\}$ are majorizing sequences and it is shown also that the tighter sequence is $\left\{s_{n}\right\}$.

TABlE 1. Comparison between the sequences $\left\{s_{n}\right\},\left\{t_{n}\right\}$ and $\left\{w_{n}\right\}$

\begin{tabular}{cccc}
\hline $\mathrm{n}$ & $\left\|s_{n+1}-s_{n}\right\|$ & $\left\|t_{n+1}-t_{n}\right\|$ & $\left\|w_{n+1}-w_{n}\right\|$ \\
\hline 1 & $0.0150308 \cdots$ & $0.0200411 \cdots$ & $0.0232399 \cdots$ \\
2 & $0.00197814 \cdots$ & $0.00292257 \cdots$ & $0.00446203 \cdots$ \\
3 & $0.0000890021 \cdots$ & $0.000181477 \cdots$ & $0.000339709 \cdots$ \\
4 & $4.88677 \times 10^{-7}$ & $1.53289 \times 10^{-6}$ & $4.52784 \times 10^{-6}$ \\
5 & $1.16179 \times 10^{-10}$ & $7.63675 \times 10^{-10}$ & $4.32958 \times 10^{-9}$ \\
6 & $1.66533 \times 10^{-16}$ & $3.16414 \times 10^{-15}$ & $5.45120 \times 10^{-14}$ \\
\hline
\end{tabular}

\section{Conclusion}

We present a new semilocal convergence analysis for the secant method in order to approximate a locally unique solution of a nonlinear equation in a Banach space setting. We showed that the new convergence criteria can be always weaker than the corresponding ones in earlier studies such as $[1,14,17$, 19,21-24,27,28]. Numerical examples where the old results cannot guarantee the convergence but our new convergence criteria can are also provided in this study.

Acknowledgements. The research has been supported by the research project Ref. MTM2014- 52016-C2-1-P of the Spanish Ministry of Science and Innovation and by the Universidad Internacional de La Rioja (UNIR, http://www.unir. 
net), under the Plan Propio de Investigación, Desarrollo e Innovación [20132015], research group: Matemática aplicada al mundo real (MAMUR).

\section{References}

[1] S. Amat, S. Busquier, and J. M. Gutiérrez, On the local convergence of secant-type methods, Int. J. Comput. Math. 81 (2004), no. 9, 1153-1161.

[2] S. Amat, S. Busquier, and Á. A. Magreñán, Reducing chaos and bifurcations in Newtontype methods, Abstr. Appl. Anal. 2013 (2013), Article ID 726701, 10 pages.

[3] S. Amat, S. Busquier, and M. Negra, Adaptive approximation of nonlinear operators, Numer. Funct. Anal. Optim. 25 (2004), no. 5-6, 397-405.

[4] S. Amat, Á. A. Magreñán, and N. Romero, On a two step relaxed Newton-type methods, Appl. Math. Comput. 219 (2013), no. 24, 11341-11347.

[5] I. K. Argyros, A unifying local-semilocal convergence analysis and applications for twopoint Newton-like methods in Banach space, J. Math. Anal. Appl. 298 (2004), no. 2, 374-397.

[6] Math. J. 55(130) (2005), no. 1, 175-187.

[7] — Convergence and Applications of Newton-Type Iterations, Springer-Verlag Publ., New-York, 2008.

[8] Weaker conditions for the convergence of Newton's method, J. Complexity 28 (2012), no. 3, 364-387.

[9] I. K. Argyros, D. González, and Á. A. Magreñán, A semilocal convergence for a uniparametric family of efficient secant-like methods, J. Function Spaces 2014 (2014), Article ID 467980, 10 pages.

[10] W. E. Bosarge and P. L. Falb, A multipoint method of third order, J. Optimization Theory Appl. 4 (1969), 156-166.

[11] J. E. Dennis, Toward a unified convergence theory for Newton-like methods, in Nonlinear Functional Anal. and Appl. (Proc. Advanced Sem., Math. Res. Center, Univ. of Wisconsin, Madison, Wis., 1970) pp. 425-472 Academic Press, New York, 1971.

[12] M. García-Olivo, El método de Chebyshev para el cálculo de las raíces de ecuaciones no lineales, (PhD Thesis), Servicio de Publicaciones, Universidad de La Rioja, 2013.

[13] J. M. Gutiérrez, Á. A. Magreñán, and N. Romero, On the semilocal convergence of Newton-Kantorovich method under center-Lipschitz conditions, Appl. Math. Comput. 221 (2013), 79-88.

[14] M. A. Hernández, M. J. Rubio, and J. A. Ezquerro, Secant-like methods for solving nonlinear integral equations of the Hammerstein type, J. Comput. Appl. Math. 115 (2000), 245-254.

[15] Solving a special case of conservative problems by Secant-like method, Appl. Math. Comput. 169 (2005), no. 2, 926-942.

[16] L. V. Kantorovich and G. P. Akilov, Functional Analysis, Pergamon Press, Oxford, 1982.

[17] P. Laasonen, Ein überquadratisch konvergenter iterativer algorithmus, Ann. Acad. Sci. Fenn. Ser I 450 (1969), 1-10.

[18] Á. A. Magreñán, Estudio de la dinámica del método de Newton amortiguado, PhD Thesis, Servicio de Publicaciones, Universidad de La Rioja, 2013.

[19] J. M. Ortega and W. C. Rheinboldt, Iterative Solution of Nonlinear Equations in Several Variables, Academic Press, New York, 1970.

[20] A. M. Ostrowski, Solution of equations in Euclidian and Banach Spaces, Academic Press, New York, 1972.

[21] F. A. Potra, An error analysis for the secant method, Numer. Math. 38 (1982), no. 3, $427-445$. 
[22] $\ldots$, Sharp error bounds for a class of Newton-like methods, Libertas Math. 5 (1985), $71-84$.

[23] F. A. Potra and V. Pták, Nondiscrete Induction and Iterative Processes, Pitman, New York, 1984

[24] J. W. Schmidt, Untere Fehlerschranken für Regula-Falsi Verhafren, Period. Math. Hungar. 9 (1978), no. 3, 241-247.

[25] A. S. Sergeev, The method of Chords, Sibirsk, Mat. Ž. 11 (1961), 282-289.

[26] S. Ulm, A majorant principle and the method of secants, Izv. Akad. Nauk Eston. SSR, Ser. Fiz.-Mat. 13 (1964), 217-227.

[27] T. Yamamoto, A convergence theorem for Newton-like methods in Banach spaces, Numer. Math. 51 (1987), no. 5, 545-557.

[28] M. A. Wolfe, Extended iterative methods for the solution of operator equations, Numer. Math. 31 (1978), no. 2, 153-174.

Á. Alberto Magreñán Escuela De Ingeniería Universidad Internacional De La Rioja 26005, Logroño, La Rioja, Spain

E-mail address: alberto.magrenan@unir.net

IOANNIS K. ARgYros

Department of Mathematics Sciences

CAmeron University

LAWTON, OK 73505, USA

E-mail address: iargyros@cameron.edu 